\title{
Revolución científica de la salud familiar en Chile ${ }^{1}$
}

Denisse Parra Giordano ${ }^{2}$

Institución: Universidad de Chile

\section{ENSAYO}

\section{COMO CITAR}

Parra, D. (2016). Revolución científica de la salud familiar en Chile. Rev. Enfermería Actual en Costa Rica, 31, 1-16. DOI: http://dx.doi.org/10.15517/revenf.v0i31.23159

\section{RESUMEN}

Introducción. El objetivo de la presente reflexión es analizar el cambio de modelo de atención en salud en Chile. Desarrollo. Se analiza el marco conceptual de la estructura de las Revoluciones Científicas de Thomas Kuhn, mediante un procedimiento que compare el proceso de cambio vivido en el país desde un paradigma de salud tradicional con un marcado principio biomédico, hasta la adopción del Modelo de Atención Integral de Salud Familiar con un enfoque biopsicosocial, el cual considere el proceso de transformación de los diferentes actores, principalmente de la Enfermería Comunitaria.

Conclusión. La mudanza de modelo de salud en Chile corresponde a una Revolución Científica. Esta visión de salud es un pilar en la atención de enfermería y en su rol como profesional del equipo de salud comunitario.

Palabras clave: enfermería-en-salud-comunitaria, filosofía-en-Enfermería, salud-familiar.

\footnotetext{
${ }^{1}$ Fecha de recepción: 4 de febrero, 2016

Fecha de aceptación: 5 de mayo, 2016

${ }^{2}$ Enfermera. Profesor Asistente Departamento de Enfermería, Universidad de Chile. Chile. Correo electrónico: denisseparrag@gmail.com
} 


\section{Scientific Revolution of family health in Chile $^{1}$}

Denisse Parra Giordano ${ }^{2}$

Institution: University of Chile

\section{ESSAY}

\section{CITED}

Introduction. The aim of this reflection is to analyze the model change health care in Chile.

Development. The conceptual framework of The Structure of Scientific Revolutions by Thomas Kuhn is analyzed by a method that compares the process of change lived in the country from a paradigm of traditional health with a strong biomedical first, until the adoption of the Model of Comprehensive Care Family health with a biopsychosocial approach, which considers the transformation process of the different actors, mainly from the Community Nursing.

Conclusion. Moving health model in Chile corresponds to a Scientific Revolution. This vision of health is a pillar in nursing care and its role as a professional community health team.

Key words: community-health-nursing, family-health, philosophy-in-nursing.

\footnotetext{
${ }^{1}$ Date of receipt: February 4, 2016

Date of acceptance: May 5, 2016

${ }^{2}$ Nurse. Assistant Professor Department of Nursing, University of Chile. Chile. E-mail: denisseparrag@gmail.com
} 


\title{
Revolução Científica de saúde da familia no Chile ${ }^{1}$
}

Denisse Parra Giordano ${ }^{2}$

Instituição: Universidade do Chile

\author{
ENSAIO
}

\section{CITAÇÃO}

Parra, D. (2016). Revolução Científica de saúde da familia no Chile. Rev. Enfermería Actual en Costa Rica, 31, 1-16. DOI: http://dx.doi.org/10.15517/revenf.v0i31.23159

Introdução. O objetivo da presente reflexão é analisar a mudança de modelo de atenção na saúde no Chile.

Desenvolvimento. O marco conceitual a estrutura das Revoluções Científicas de Thomas Kuhn, mediante um procedimento que compare o processo de mudança vivido no país desde um paradigma da saúde tradicional com um marcado princípio biomédico, até a adoção do Modelo de Atenção Integral de Saúde Familiar com um enfoque biopsicosocial, o qual considera o processo de transformação dos diferentes autores, principalmente da Enfermagem Comunitária.

Conclusões Conclui-se que a mudança do modelo de saúde no Chile corresponde a uma Revolução Científica. Esta visão de saúde é um pilar na atenção da enfermagem e em seu papel como profissional da equipe de saúde comunitária.

Palavras-chave: enfermagem-na-saúde, comunitária-saúde-familiar, filosofía-em-enfermagem.

\footnotetext{
${ }^{1}$ Data de recepção: 4 de fevereiro de 2016

Data de aceitação: 5 de maio de 2016

${ }^{2}$ Enfermeira. Professor Assistente do Departamento de Enfermagem da Universidade de Chile. Chile. Correio eletrônico: denisseparrag@gmail.com
} 


\section{Revista Electrónica Enfermeria Actual en costa Rica}

\section{INTRODUCCIÓN}

Chile es un país largo y angosto, que se extiende dese la Cordillera de Los Andes y el Océano Pacífico al suroeste de América del Sur; presenta particularidades geográficas ya que posee territorio en la Polinesia con la Isla de Pascua, en la Antártida y en el continente sudamericano (Universidad de Chile, s. f.).

Dicho país no es conocido solo por su riqueza y encanto natural, sino por la importancia de la salud para el Estado chileno que, desde comienzos del siglo XIX, ha erigido diferentes instituciones cuyo objetivo pretende estructurar el sector salud en Chile, tal es el caso de la Junta de Vacunas (1808) (Gobierno de Chile, s. f.), el Ministerio de Salud (1959), y el Sistema Nacional de Servicios de Salud (1979), las cuales han contribuido a la descentralización del Sistema de Salud (Gobierno de Chile, s. f.).

A nivel internacional, Chile se adhiere a la declaración de Alma Ata de 1978, en la que se subraya la importancia de la atención primaria de salud como estrategia para alcanzar un mejor nivel de salud de los pueblos. Respecto de los sistemas de salud de países desarrollados inscritos en la Organización para la Cooperación y el Desarrollo destaca que aquellos con una atención primaria "más fuerte" logran estándares de salud mejores para sus ciudadanos, altos niveles de satisfacción usuaria y per cápita menor en salud (Starfield, Shi y Macinko, 2005).

Partiendo de que la atención primaria necesita un enfoque familiar con dominio poblacional; es decir, un trabajo con todas las familias de una comunidad (García-Huidobro, 2010; Püchel, Soto, Cerda y Rojas, 2012), en el año 1993 con el objetivo de desarrollar la atención primaria en Chile se inició la transformación de los Consultorios a Centros de Salud (CES) (Depaux, Campodónico, Ringeling y Segovia, 2008). Siguiendo con este desenvolvimiento en 1998 se comienza la transformación de CES a Centro de Salud Familiar (CESFAM), proceso en el que se agregó a los ejes ya existentes, los de la familia como unidad de análisis y como estrategia inherente, incorporándose la visita domiciliaria integral y la consejería, lo cual refuerza la perspectiva familiar al intervenir no solamente al individuo, sino a la familia de este, tanto en lo concerniente a la valoración por medio de la aplicación de instrumentos como el genograma o APGAR familiar en la visita domiciliaria, como en la posterior intervención a domicilio que también incluye a los integrantes; es decir, sin limitarse únicamente al caso índice (Depaux, et al, 2008; Dois, et al., 2013).

En el desarrollo de las visitas domiciliarias, el profesional de enfermería cumple un rol muy importante al tener -desde el pregrado- la capacitación para realizar una visita domiciliaria integral y un estudio de familia, realizando el proceso de enfermería familiar completo. Se reconoce al enfermero/a como parte del equipo de salud en Chile, siendo parte básica en la composición de los equipos de cabecera en los CESFAM (Depaux, et al, 2008; Dois et al., 2013).

En el año 2001, con la Reforma del sistema de Salud, el Modelo de Atención Integral con Enfoque Familiar y Comunitario fue legalmente aprobado como la nueva manera de atender, además de que se estableció las bases de una gestión en torno a redes asistenciales (Depaux, et al, 2008; Dois, et al, 2013). 


\section{Revista Electrónica Enfermeria Actual en costa Rica}

Este cambio de paradigma de atención de salud que legalmente comenzó hace 20 años implicó para los equipos de salud y el profesional de enfermería un proceso de cuestionamiento e implementación mucho mayor, que continúa hasta la fecha. Durante todo el proceso, la enfermería ha estado presente contribuyendo de manera significativa desde su formación holística y preparación en el área comunitaria.

Para analizar este proceso se utilizará como marco conceptual el planteado por Tomas Kuhn en su libro la "Estructura de las Revoluciones Científicas", el cual se refiere a la revolución científica, entendida como el proceso de cambio de paradigma de atención de salud desde una perspectiva biomédica a una que incluya la salud integral familiar con el fin de identificar las etapas que conllevan el surgimiento de una nueva teoría, del cambio de una antigua por la nueva.

\section{DESARROLLO}

\section{Contexto histórico en Chile}

En Chile, en la década de los 90, ocurrieron cambios culturales y sociales a raíz del perfil epidemiológico de la época, respecto de que disminuyeron las dolencias infecciosas pero aumentaron las enfermedades cardiovasculares para las que -el modelo biomédico de la época- no tenía respuesta (Depaux, et al, 2008; Dois, et al., 2013; Püchel, et al., 2012; Sepúlveda, 2012). Frente a dicha realidad, Chile comenzó la instauración de un modelo de salud integral (Depaux, et al., 2008; Püchel, et al., 2012; Gobierno de Chile, 2005; Sepúlveda, 2012), fundamentado en los valores del derecho, la equidad y la solidaridad, y en los principios de accesibilidad, oportunidad, calidad y uso eficiente de recursos que se ve refleja en los tres niveles de atención de salud en el país a través de diferentes mecanismos (Gobierno de Chile, 2005; Sepúlveda, 2012).

Fue entonces cuando se instauró un sistema de atención integral de salud centrado en la persona y familia, a cargo de equipos de cabecera para realizar un trabajo participativo que mejorara el nivel de salud de toda la comunidad (Dois, et al., 2013; Püchel, et al., 2010), cuya prioridad son actividades de promoción de la salud, prevención de la enfermedad y de brindar herramientas de autocuidado para las personas, con enfoque territorial y eje de intervención las personas, familias y comunidad (Dois, et al., 2013; Rojas, 2010). Dicho abordaje concuerda con la esencia del profesional de enfermería, el cual se centra en la persona y reconoce la responsabilidad de los individuos y familia en su propio cuidado de salud (Caballero, Becerra y Ibarra, 2010).

Además, de la visión integral, la transformación del centro de salud a centro de salud familiar se enmarcó en una política nacional de reforma al sistema sanitario que releva a la Atención Primaria de Salud (APS) como un eje fundamental para el logro de una mejoría en la calidad de vida de la población (Depaux, et al., 2008; Rojas, 2010); es decir, se esperaba que la mirada de atención de salud chilena no solamente integre nuevos saberes como la consejería o visita domiciliaria integral, sino que mudara su visión del cuidado en salud con un foco en la familia y mirada biopsicosocial, que reforzara el modelo de autocuidado de enfermería de Dorotea Orem (Caballero, et al., 2010).

\section{Implementación modelo de salud familiar}




\section{Revista Electrónica Enfermeria Actual en costa Rica}

En el año 2012 se cumplieron 50 años desde la publicación del libro La Estructura de las Revoluciones Científicas de Kuhn (1962) (Artigas, 2009; Assis, 1993; Briceño, 2009), quien se interrogó acerca del uso desmedido de la lógica y la idea del progreso continuo (Briceño, 2009), respecto de lo que manifiesta que el conocimiento científico no es el resultado de la acumulación de saberes, sino de los cambios de paradigmas, en otras palabras, de la adopción de nuevos enfoques, conceptos y compromisos por la comunidad científica (Artigas, 2009; Briceño, 2009; Sepúlveda, 2012).

De acuerdo con el planteamiento de Kuhn, existen tres instantes claves en el desarrollo del conocimiento: el primero la ciencia normal, después la presencia de las anomalías, y por último la crisis y el desenvolvimiento de esta (Artigas, 2009; Assis, 1993; Briceño, 2009). En la etapa de ciencia normal, se remarca la importancia de las comunidades para conocer estas teorías y aplicarlas (Artigas, 2009; Assis, 1993; Briceño, 2009). Al respecto, en Chile siempre se ha trabajado con un marco legal en salud entregado desde el Ministerio de Salud, y supervisado a nivel nacional por las secretarias ministeriales en cada región y, además, por los servicios de salud (actualmente 29 a nivel nacional) (Parra y Andres, 2013), de modo que el modelo de salud utilizado sea conocido por todas las personas que trabajan en esta área e impartido en los centros educacionales de formación de profesionales del área de salud. Del mismo modo, en los últimos años también se ha trabajado en incorporar a los usuarios como pieza fundamental en el conocimiento y aplicación de la teoría de salud que aplicamos a nivel nacional.

Respecto de los conocimientos que comparte una comunidad, Kuhn propone el término matriz disciplinar, el cual se estructura con elementos ordenados y cada uno precisa de una especificación posterior (Assis, 1993; Briceño, 2009). Dicha matriz, utilizada por siglos, corresponde al modelo biomédico en salud, desarrollado a partir del siglo XVII y solventa la necesidad del ser humano en cuanto a construir creencias o teorías que le permitan ordenar el mundo de una manera tranquilizadora, dada su poca tolerancia a la incertidumbre.

El modelo biomédico ha permitido grandes avances en el conocimiento y desarrollo tecnológico, pero pierde validez científica cuando deja fuera las variables humanas (Depaux, et al, 2008), razón por la que también puede identificarse con lo planteado por Kuhn, puesto el modelo biomédico, usado por tantos años en salud, emplea la lógica y se valida mediante el método científico, sin dejar por fuera variables tan importantes para el autor, como el contexto histórico y social para la validez y aceptación de una teoría.

Respecto de la segunda etapa, la anomalía, existe una falta de respuesta del paradigma actual a la resolución de problemas (Artigas, 2009; Assis, 1993; Briceño, 2009). El modelo biomédico comenzó a ser insuficiente para el equipo de salud y el profesional de enfermería, dado que la atención -desde esta teoría- se basa en una concepción patologista y reduccionista (Depaux, et al., 2008), centrada solamente en un órgano o dolencia afectada de la persona; por ejemplo, en los hospitales las personas son llamadas por la patología que poseen o por el número de la cama que ocupan sin que se conozca su nombre.

Por otra parte, valora los datos objetivos mediante la división mente y cuerpo (Depaux, et al., 2008) y otorga mayor importancia a los valores entregados por la tecnología, como en el caso de la tomografía axial computacional respecto de la información que brinda la propia persona doliente, así como es el nivel de intensidad del dolor que padece o la ubicación exacta de este. También, tiene como fin último la curación, hace diagnósticos sobre la base de conocimientos y considera como recursos para el tratamiento elementos externos 


\section{Revista Electrónica Enfermeria Actual en costa Rica}

(Depaux, et al., 2008), como por ejemplo lo que sucede con los fármacos de alto precio, sin que se considere el nivel socioeconómico del usuario o como cuando se indica procedimientos con elevada dificultad técnica sin evaluar la presencia de familiares o amigos en el proceso de salud-enfermedad de la persona.

Tales principios de salud delimitaban las políticas públicas que se venían realizando; no obstante, el personal sociosanitario, en especial el de enfermería, evidenciaba que esta mirada no era suficiente para intervenir efectivamente a un usuario, sin lograr la compensación de una patología y, más aún, la adherencia a este modelo de intervención probado científicamente. Un ejemplo es el caso de los usuarios con diabetes mellitus quienes eran diagnosticados desde el modelo tradicional, además de que sus intervenciones eran planificadas con medidas centradas en la patología, así como las indicaciones alimenticias, pensadas desde el ideal que lo que el usuario debe comer; en tales casos, en sus controles posteriores el usuario no logra compensar su enfermedad (incluso, a veces abandona el tratamiento), ante lo cual el equipo de salud y en especial el profesional de enfermería se da cuenta de que en el tratamiento de esta enfermedad participan otros factores como el trabajo, el nivel socioeconómico, etc., y, principalmente, la familia.

Los equipos de salud, y principalmente los profesionales de enfermería, han ido implementando pequeños remedios a las deficiencias existentes, pero estas no se adecuan a las políticas públicas. Del mismo modo, desde la dirección de salud se evidencia que las estrategias existentes no son capaces de dar respuesta a los objetivos de salud de las personas, por tanto, es necesaria la implementación de un modelo que responda a los equipos de salud y al gobierno y que redunde en la salud para las personas. Ante la situación mencionada, nace la crisis que cuestiona la validez del paradigma actual (Artigas, 2009; Briceño, 2009) y representa el momento en que se toma conciencia de la presencia de la anomalía (Briceño, 2009). Como se evidencia en los párrafos anteriores, existe no solamente la presencia de discrepancias con el modelo actual de salud en los años 80 en Chile, sino que estas divergencias son reconocidas por la comunidad de los profesionales de salud, mientras cuestionan el modelo y procuran nuevas formas de atención hacia otros países.

Como parte del cambio, en 1977, Engel propone el modelo biopsicosocial que deriva de la teoría general de sistemas, gracias a la continuidad temporal y multigeneracional que permite al equipo de salud atender en forma sistémica el proceso salud-enfermedad de los miembros de las familias (Depaux, et al., 2008; Rojas, 2010), lo cual se contrapone al modelo biomédico, centrado en la enfermedad, con un enfoque causa-efecto, mientras que el biopsicosocial aborda a la persona como parte de una familia y comunidad, desde el que se establece un enfoque relacional (Depaux, et al., 2008). En la práctica, el modelo biomédico atiende a nivel individual a los pacientes y con un solo profesional, mientras el modelo de salud familiar brinda la atención a nivel familiar y brinda una atención desde un equipo de salud (Depaux, et al., 2008).

Kuhn plantea que la crisis no es solamente un instante de búsqueda de conocimientos (Briceño, 2009), es decir, de implantar un nuevo modelo en vez del anterior, sino de permitir intercambios de puntos de vista y quiebres en el conocimiento establecido, en los que se debe asimilar esta crisis (Briceño, 2009), de modo que las personas implicadas conversan sobre su marco conceptual, compartiendo ideas y puntos de vista, y comienzan a construir un nuevo modelo de salud que no solamente responde a las necesidades de salud de las personas, sino a faltas en la formación del equipo de salud, de la forma de relacionarse entre el equipo y las personas y de incorporarse a la comunidad, entre otras. Una vez que se da lo anterior, se desenvuelve la ciencia extraordinaria (Artigas, 2009; Briceño, 2009), en el que la adopción del nuevo paradigma implica una postura reflexiva, lograda 


\section{Revista Electrónica Enfermeria Actual en costa Rica}

solo por medio del reconocimiento de las anomalías y el proceso de crisis (Briceño, 2009), tal como se ha desarrollado anteriormente.

Este nuevo paradigma de salud, llamado biopsicosocial, se enfoca en la persona, en sus necesidades y su contexto histórico y social, entrega un enfoque familiar, propone una mayor participación de la comunidad, continuidad de los cuidados, con mayor acento en la prevención y promoción, y presenta para el equipo de salud una atención basada en el trabajo en equipo (Briceño, 2009; Depaux, et al., 2008; Gobierno de Chile, 2005). Además, la implementación del trabajo a través del CESFAM es un cambio de una cultura organizacional tradicional a una en la que se ha desarrollado la capacidad de escuchar al otro, de centrarse en el propósito o fin común de la red; se entiende como el espacio donde se establece una relación de continuidad entre un equipo de salud y su población, relación caracterizada por la confianza y vínculos de afectividad y respeto mutuo, para el logro de un mayor bienestar de familias y comunidad (Depaux, et al., 2008).

Tal como refiere Kuhn, la crisis se cierra cuando la teoría paradigmática logra ajustarse, de modo que lo anómalo se torna parte de lo esperado (Briceño, 2009). El equipo ministerial de salud chileno plantea que ha sido necesario cambiar desde un enfoque centrado en problemas biomédicos específicos de los individuos a una programación enfocada en la persona, su familia y comunidad, es decir, las orientaciones programáticas emanadas del Ministerio de Salud han ido progresivamente incorporando mayor cantidad de elementos centrales del nuevo paradigma como son la mirada del ciclo vital, familia y comunidad, entre otros (Depaux, et al., 2008).

Otro aspecto muy importante que menciona Kuhn, es la idea de que el nuevo paradigma no puede compararse con el anterior, es decir, plantea la inconmensurabilidad de los paradigmas (Artigas, 2009; Briceño, 2009) aunque, lamentablemente, este concepto en la práctica no es aplicado ya que de manera constante el profesional de salud compara ambos modelos, intentando establecer la superioridad de uno por sobre el otro para su aplicación; entonces, no se comprende este principio de incompatibilidad entre los significados de los marcos conceptuales del nuevo y antiguo paradigma, por lo tanto, la imposibilidad de confrontarlos (Artigas, 2009; Briceño, 2009).

El último concepto que aporta el autor es el de conversión, el cual explica cómo los científicos adoptan un nuevo paradigma; sin embargo, esta aceptación no puede forzarse (Assis, 1993). Como el autor plantea, la adopción de un nuevo paradigma no debe ser solamente algo impuesto y entendido como necesario por un grupo de personas o autoridades que dicten la norma, sino que es un proceso en el cual es necesario que exista un quiebre con el modelo actual, de tal forma que se busque un modelo capaz de dar respuesta a las necesidades, motivo por el que es tan importante el reconocimiento del nuevo paradigma por las comunidades científicas (Briceño, 2009).

En Chile, dicho proceso ha sido paulatino y heterogéneo en sus grados de desarrollo (Püchel, et al., 2012): desde el comienzo de transformación de los consultorios a CES transcurrieron ocho años para que se dictara la norma de instauración del uso del nuevo modelo, el cual aún no ha sido aceptado en su totalidad por los trabajadores del equipo de salud; no obstante, ha sido el profesional de enfermería quien lo ha incorporado desde el inicio de su formación en Chile en conjunto con las enfermeras sociosanitarias, quienes han sido reconocidos como los encargados de programas y directores de los centros. Sin embargo, como el mismo Kuhn menciona, es necesario que aquellas personas que creen firmemente en el paradigma antiguo y no percibieron las anomalías y 


\section{Revista Electrónica Enfermeria Actual en costa Rica}

que, por tanto, no entraron en crisis ni aceptan el nuevo paradigma, fallezcan para que el nuevo modelo sea aceptado por toda la comunidad.

\section{CONCLUSIONES}

Las revoluciones científicas son un efecto del desarrollo de la ciencia normal, las cuales dan lugar a un nuevo paradigma que inicia una nueva era de ciencia normal (Artigas, 2009). Como Kuhn establece, el desarrollo de la ciencia presenta una estructura claramente definida, aplicable para el desenvolvimiento de nuevos paradigmas no solamente en el área de las ciencias naturales, sino en el crecimiento de las ciencias de la salud.

Desde el rol de enfermería, la implementación del nuevo modelo de atención biopsicosocial, ha estado presente en la enfermería desde antes de su llegada a Chile, debido a que la enfermera sanitaria incluía en su valoración el contexto biopsicosocial del usuario. De esta forma este modelo es un pilar en la atención de enfermería y en su rol como profesional del equipo de salud.

Por último, como profesional de salud, es enriquecedor comprender cómo el proceso de cambio de paradigma de modelo de atención sufrido en nuestro país, obedece a una estructura desarrollada por Kuhn hace medio siglo y brinda las herramientas para vislumbrar cómo el paradigma actual también se tornará el antiguo en un nuevo proceso. Al respecto, es importante retratar la evolución chilena, en aras de aportar en la implementación de este modelo en otros lugares a nivel mundial.

\section{REFERENCIAS BIBLIOGRÁFICAS}

Artigas, M. (2009). Filosofía de la ciencia (Segunda). Navarra, España: Ediciones Universidad de Navarra, S.A. (EUNSA).

Assis, J. (1993). Kuhn e as ciências sociais. Estudos Avançados, 7(19), 133-164. http://doi.org/10.1590/S0103$\underline{40141993000300004}$

Briceño, T. (2009). El paradigma científico y su fundamento en la obra de Thomas Kuhn. Tiempo y Espacio, 19 (52), 285-297.

http://dialnet.unirioja.es/servlet/articulo?codigo=3579139

Caballero, E., Becerra, R., Ibarra, A. (2010). Aplicación del Proceso de Enfermería en algunos modelos teóricos. En: Caballero M., Becerra S, Hullin L. Proceso de enfermería e informática para la gestión del cuidado. Santiago, Chile: Mediterráneo.

Depaux, R., Campodónico, L., Ringeling, I., y Segovia, I. (2008). En el camino a centro de salud familiar. Santiago, Chile: Ministerio de Salud. http://buenaspracticasaps.cl/wp-content/uploads/2014/07/MINSAL2008-En-el-camino-a-Centro-de-Salud-Familiar.pdf 


\section{Revista Electrónica Enfermeria Actual en costa Rica}

Dois, A., Ojeda, I., Vargas, I., Larrea, M., Quiroz, M., Rodríguez, M. et al. (2013). Orientaciones para la implementación del modelo de atención integral de salud familiar y comunitaria. Santiago, Chile: Ministerio de Salud. http://web.minsal.cl/portal/url/item/e7b24eef3e5cb5d1e0400101650128e9.pdf

García, D. (2010). Enfoque familiar en la Atención Primaria: Una propuesta para mejorar la salud de todos. Revista médica de Chile, 138(11), 1463-1464.

http://www.scielo.cl/scielo.php?script=sci_arttext\&pid=S0034-98872010001200019

Gobierno de Chile, Ministerio de Salud. (s. f.). Historia del MINSAL (1a parte). Recuperado 2 de febrero de 2016, a partir de http://www.minsal.gob.cl/portal/url/page/minsalcl/g_conozcanos/g_mision_vision/hist_inicios_mision_visi on.html

Gobierno de Chile, Ministerio de Salud. (s. f.). Historia del MINSAL (2a parte). Recuperado a partir de http://web2.minsal.cl/historia_minsal_2

Gobierno de Chile, Ministerio de Salud. Subsecretaría de Redes Asistenciales. División de Gestión de la Red Asistencial. (2005). Modelo de Atención Integral de Salud. Serie Cuadernos Modelo de Atención $N^{\circ} 1$. Santiago, Chile.

Parra, G., Andres, F. (2013). Trabajo de la enfermera docente en Chile: una reflexión personal. Horiz Enferm, 24(1), 83-89.

Püchel, K., Soto, G., Cerda, P., Rojas, P. (2012). Modelo de atención en Salud Familiar: principios, fundamentos e implementación. En: Dois, C. A., Montero, O. L. Manual de Atención de Familias para Profesionales de la Salud (Primera Edición). Santiago, Chile.: Ediciones UC.

Rojas, V. (2010). Modelo de atención integral desde un enfoque familiar y comunitario. En: Caballero, M., Becerra, S, Hullin, L. Proceso de enfermería e informática para la gestión del cuidado. Santiago: Mediterráneo.

Sepúlveda, A. (2012). La política pública de salud a nivel local. Análisis de la transformación del Centro de Salud Familiar Isabel Jiménez Riquelme de Tirúa, Biobío, Chile. Revista Chilena de Administración Pública, 19, 67 a 103. http://www.nuevosfoliosbioetica.uchile.cl/index.php/REGP/article/viewArticle/25860

Starfield, B., Shi, L., Macinko, J. (2005). Contribution of primary care of health systems and health. Milbank Quaterly, 83(3), 457-502.

Universidad de Chile. (s. f.). Acerca de Chile. Tierra de Contrastes. Recuperado 4 de febrero de 2016, a partir de http://www.uchile.cl/portal/presentacion/la-u-y-chile/acerca-de-chile 